\title{
Blind oral intubation: a forgotten technique of intubation
}

\author{
Magboul MA Magboul* \\ Clinical Assistant Professor of Anesthesia, University of Iowa, College of Medicine, Iowa City, USA
}

\section{Case report}

A 17-year old male patient ASA 1 presented for vitrectomy of his right eye. His medical record showed no significant medical concerns apart from his eye problems. However he was documented to have difficult intubation in two previous anesthetics occasions.

His airway assessment scored more than 8 on Magboul Scale [1]; hence we predicted difficulty in his intubation again.

We decided to deal with his intubation problem by using a C- Mac video laryngoscope.

Anesthesia was induced with $2 \mathrm{mg}$ of midazolam, 100 micrograms of Fentanyl, $200 \mathrm{mg}$ of Propofol, and $100 \mathrm{mg}$ of suxamethonium, uneventfully.

When the CRNA picked up the C-Mac blade to intubate we discovered that the blade provided was a pediatric size blade and would not behelpful in intubating this patient. We then called the work room for a larger adult size blade. The estimated time for blade arrival was 3-5 minutes.

I told the CRNA that while we are waiting I would demonstrate to him an old technique for difficult intubation known as blind oral intubation.

While standing on the left side of the patient I held the tube with my right hand while manipulating the Larynex with my left hand to meet the tip of the tube at the vocal cord.

The first attempt took 10 seconds, and was oesophageal intubation. The tube was removed and the stylet angle near the end was reduced, a second attempt was successful and took 10 seconds to achieve a successful endo tracheal intubation before the adult blade arrival.

\section{Discussion}

Blind oral and nasal intubations are old techniques used successfully in the past for difficult intubation before the arrival of fiberoptics and video laryngoscopes (Figure 1).

It is a pity that not all institutes continue to train their juniors to master these old techniques now a days.

Digital intubation allows intubation to be performed without a laryngoscope or a view of the larynx and may be performed with or without a bougie [2].

It can be used in cramped environment (e.g. patient trapped in vehicle), copious oral fluids (e.g. large amount of blood or vomitus in oral cavity, obscuring visualization with a laryngoscope), inability to visualize vocal cords with laryngoscope, severe head/ neck trauma requiring immobilization of cervical spine.

There are several techniques and methods among them, blind digital intubation without a bougie technique of bougie-assisted blind digital intubation from Rich in 2008 [3] Or Stewart's tactile orotracheal intubation [4], Vacanti's blind oral intubation [5], or the simple Magboul technique that I used in this case.

By standing on the left side of the patient I hold the tube with my right hand while externally manipulating the Larynex with my left hand to meet the tip of the tube at the vocal cord.

In other occasions the BURP (The Backward, Upward to the Right Pressure) manoeuver established by Knell 1993 is also helpful in making the two ends meets [6].

A study that compared the BURP technique with bimanual manipulation in cadavers [7], it was found that bimanual manipulation improved the percentage of glottic opening more than BURP or cricoid. However that was for direct laryngoscopy and not for blind oral intubation.

Bimanual manipulation is putting external pressure on the thyroid cartilage and moving it while inserting the tip of the laryngoscope into the vallecula. The effect is that it assists the tip of the blade in reaching deeper into the vallecula and thus assists in elevating the epiglottis.

The advantages of oral blind intubation is that it is fast in experienced hands, there is no requirement for optimal positioning with minimal c-spine movement for trauma patients. It is also ideal for those predicted to be difficult airway, and can be used if copious secretions

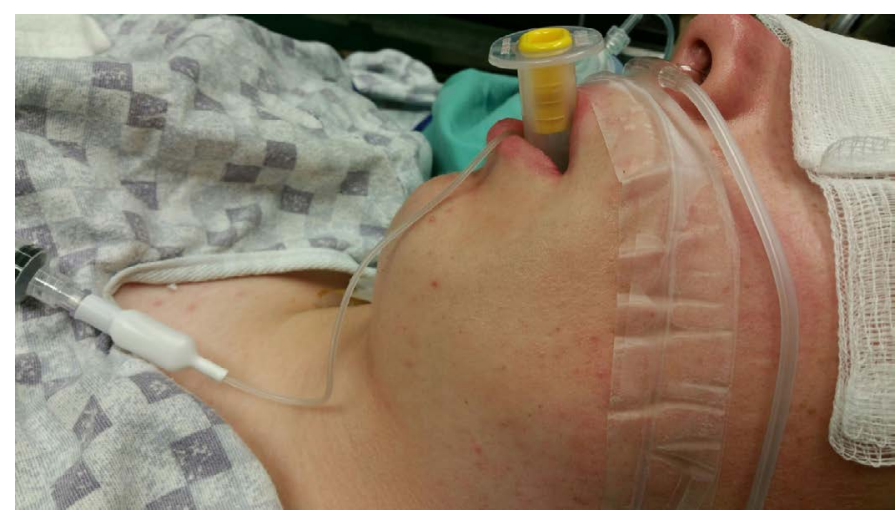

Figure 1. Patient lateral view after successful blind oral intubation.

Correspondence to: Magboul MA Magboul, MD, FFARCSI, Clinical Assistant Professor Anesthesia, University of Iowa, college of Medicine, Department of Anesthesia-6JCP, 200 Hawkins Drive, Iowa City, Iowa 52242-1009, USA, Tel: (319) 3568497 (Office); Tel: (319) 356-2633 (Department); Tel: (319) 3567471 (Sec. office); Fax: (319) 3562940 (Department); E-mail: m-magboul@uiowa.edu

Received: June 20, 2016; Accepted: July 09, 2016; Published: July 12, 2016 
or blood in airway when you cannot visualize the known landmarks.

However the disadvantages of oral blind intubation are requirement of training, the risks to operator fingers trauma from patient's teeth. Airway trauma, and may benefits operators with long, slender fingers.

In conclusion, Blind oral tracheal intubation can be successfully performed in a safe and effective manner after appropriate teaching of the technique. There is a collection of aid devices for blind oral intubation available in the market as well.

Blind oral intubation helped us in intubating our patient successfully while waiting for the expensive video laryngoscope.

\section{References}

1. Magboul MM (2004) The Dilemma of Airway Assessment and Evaluation. The Internet Journal of Anesthesiology 10: 1.
2. Hardwick WC, Bluhm D (1984)Digital intubation. J Emerg Med 1: 317-20.

3. Rich JM (2008) Successful blind digital intubation with a bougie introducer in a patient with an unexpected difficult airway. Proc (Bayl Univ Med Cent) 21: 397-9. [Crossref]

4. Stewart RD (1984) Tactileorotracheal intubation. Ann Emerg Med 13: 175-8.[Crossref]

5. Vacanti CA, Roberts JT (1992) Blind oral intubation: the development and efficacy of a new approach. J Clin Anesth 4: 399-401. [Crossref]

6. Young SE, Miller MA, Crystal CS, Skinner C, Coon TP (2006) Is digital intubation an option for emergency physicians in definitive airway management? Am J Emerg Med 24: 729-32.[Crossref]

7. Levitan RM, Kinkle WC, Levin WJ, Everett WW (2006) Laryngeal view during laryngoscopy: a randomized trial comparing cricoid pressure, backward-upwardrightward pressure, and bimanual laryngoscopy. Ann Emerg Med 47: 548-55. [Crossref]

Copyright: (O2016 Magboul MMA. This is an open-access article distributed under the terms of the Creative Commons Attribution License, which permits unrestricted use, distribution, and reproduction in any medium, provided the original author and source are credited. 